\title{
Structures of $\mathrm{Cu}_{n}^{+}(n=3-10)$ Clusters Obtained by Infrared Action Spectroscopy
}

Olga V. Lushchikova, ${ }^{\dagger}$ Douwe M. M. Huitema, ${ }^{\dagger}$ Pablo López-Tarifa, ${ }^{\ddagger}$ Lucas Visscher, ${ }^{\ddagger \odot}$

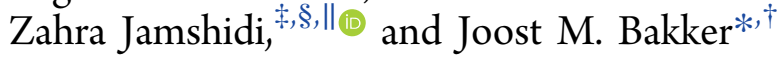

${ }^{\dagger}$ Radboud University, Institute for Molecules and Materials, FELIX Laboratory, Toernooiveld 7c, 6525 ED, Nijmegen, The Netherlands

${ }^{\ddagger}$ Division of Theoretical Chemistry, Faculty of Sciences, Vrije Universiteit Amsterdam, De Boelelaan 1083, 1081 HV Amsterdam, The Netherlands

${ }^{\S}$ Chemistry Department, Sharif University of Technology, Tehran 11155-9516, Iran

"Chemistry \& Chemical Engineering Research Center of Iran (CCERCI), P.O. Box 14968-13151, Tehran 14335-186, Iran

\section{Supporting Information}

ABSTRACT: Coinage metal clusters are of great importance for a wide range of scientific fields, ranging from microscopy to catalysis. Despite their clear fundamental and technological importance, the experimental structural determination of copper clusters has attracted little attention. We fill this gap by elucidating the structure of cationic copper clusters through infrared (IR) photodissociation spectroscopy of $\mathrm{Cu}_{n}{ }^{+}-\mathrm{Ar}_{m}$ complexes. Structures of $\mathrm{Cu}_{n}{ }^{+}(n=3-10)$ are unambiguously assigned based on the comparison of experimental IR spectra in the $70-280 \mathrm{~cm}^{-1}$ spectral range with spectra calculated using density functional theory. Whereas $\mathrm{Cu}_{3}{ }^{+}$and $\mathrm{Cu}_{4}{ }^{+}$are planar, starting from $n=5, \mathrm{Cu}_{n}{ }^{+}$ clusters adopt $3 \mathrm{D}$ structures. Each successive cluster size is composed of its predecessor with a single atom adsorbed onto the face, giving evidence of a stepwise growth.

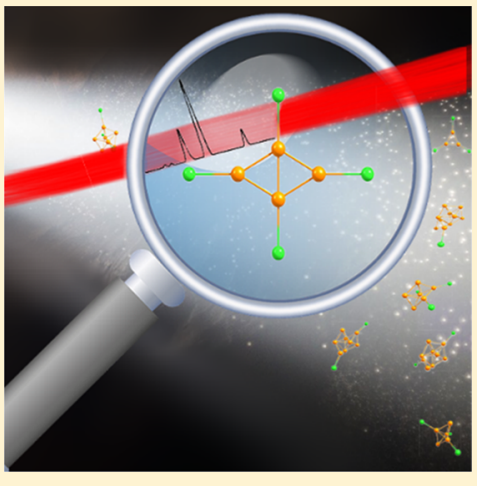

$\mathrm{T}$ ransition-metal clusters are of great interest and considerable importance to the fields of heterogeneous catalysis, solid-state physics, surface chemistry, and organometallic chemistry due to their frequently strongly sizedependent properties of magnitudes that are often not present in small molecular or bulk systems. Part of the fascination of clusters stems from their often enhanced reactivity with respect to the bulk, typically attributed to the larger number of undercoordinated atoms, involving free $\mathrm{d}$ electrons to actively participate in the bonding. The coinage metals' $(\mathrm{Cu}, \mathrm{Ag}, \mathrm{Au})$ special place in this stems from their closed $\mathrm{d}$ shell, forcing $\mathrm{s}$ electrons to become involved in bonding and often leading to much more gentle activation of feedstock molecules. ${ }^{1,2}$

Of the coinage metal clusters, gold has, not unreasonably, attracted the most attention, especially after the groundbreaking experiments by Haruta and coworkers demonstrating catalytic CO oxidation at low temperatures. ${ }^{3}$ Whereas gold certainly has laid claim to being a "special" element, the chemical importance of other coinage metal clusters has to a certain point been neglected by this proverbial gold rush. The catalytic activity of, for instance, the industrial $\mathrm{CO}_{2}$ hydrogenation catalyst has been largely attributed to copper nanoparticles on an $\mathrm{Al}_{3} \mathrm{O}_{4}$ surface with $\mathrm{ZnO}$ as a cocatalyst., It has been shown that the $\mathrm{CO}_{2}$ hydrogenation is structuresensitive, where smaller $\mathrm{Cu}$ nanoparticles exhibit larger turnover frequencies. ${ }^{6}$ Yet the mechanism for hydrogenation remains elusive, even for the very first step. Using clusters as well-defined model systems, proposed reaction pathways can be tested with high confidence, allowing much needed insight. Beside this role as a model system, clusters may also act as a catalyst under technical conditions themselves. Recently, it was shown that deposited $\mathrm{Cu}_{4}{ }^{+}$can lower the activation barrier for the methanol formation from $\mathrm{CO}_{2}{ }^{7}$ thereby illustrating the potential of nanotailored catalysts.

For both roles, however, knowledge of the clusters themselves and, in particular, of their structures, is imperative. In the model system scenario, it is of great importance to know the cluster morphology prior to exposing it to reactants, whereas for studies on deposited clusters, size-selected prior to soft landing, the influence of the substrate on the cluster geometry must be taken into account. ${ }^{8}$ Despite the fact that $\mathrm{Cu}$ clusters were among the first clusters produced, ${ }^{9}$ the available structural information is limited to photoelectron spectroscopy for anions, ${ }^{10-13}$ mass spectrometric studies using $\mathrm{H}_{2} \mathrm{O}$ as the molecular probe to reveal the number of adsorption sites, ${ }^{14}$ photodissociation spectra in the visible range, ${ }^{15,16}$ and an ionmobility mass spectrometry (IMS) study ${ }^{17}$ for cations.

Received: February 25, 2019

Accepted: April 12, 2019

Published: April 12, 2019 

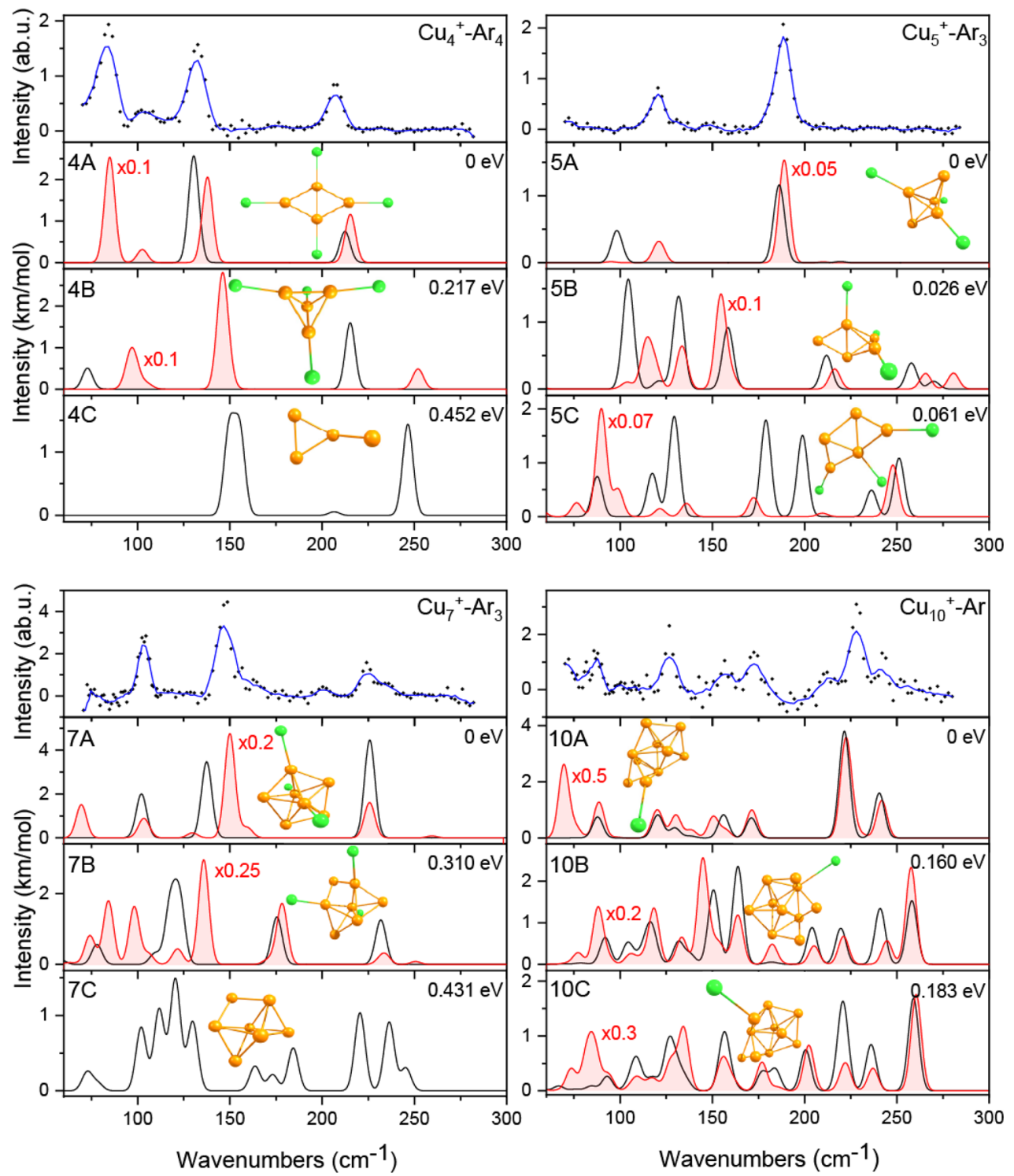

Figure 1. Comparison of IRMPD spectra of $\mathrm{Cu}_{4}{ }^{+}-\mathrm{Ar}_{4}, \mathrm{Cu}_{5}{ }^{+}-\mathrm{Ar}_{3}, \mathrm{Cu}_{7}{ }^{+}-\mathrm{Ar}_{3}$, and $\mathrm{Cu}_{10}{ }^{+}-\mathrm{Ar}$ (black dots; the blue line represents a five-point adjacent average) with calculated vibrational spectra of three low-energy isomers (black lines) and their complexes with Ar (red shading). The corresponding structures and relative energies of the bare clusters are reported above each spectrum.

Infrared multiple photon dissociation (IRMPD) spectroscopy has a solid track record in determining the molecular structure of metal clusters and can do so mass-selectively using weakly bound messenger atoms or molecules. ${ }^{18,19}$ We recorded IRMPD spectra of $\mathrm{Cu}_{n}{ }^{+}-\mathrm{Ar}_{m}(n=3-10, m=1-4)$ in a molecular beam environment using IR light produced by the free-electron laser (FEL) FELIX in the $70-280 \mathrm{~cm}^{-1}$ spectral range $^{20}$ and mass-selective detection. The experimental IR spectra for four of the cluster sizes studied are shown in Figure 1 , whereas a complete overview of spectra for $\mathrm{Cu}_{n}{ }^{+}-\mathrm{Ar}_{m}$ can be found in the Supporting Information (SI). The experimental IR spectra are complemented by spectra calculated for trial structures using density functional theory (DFT) calculations at the PBE-D3/TZVP level. Isomers for pure copper clusters as well as clusters complexed with argon are considered to assess the role of the messenger.

Complexation of copper clusters with $\mathrm{Ar}$ atoms is strongly influenced by temperature and Ar concentration; the mass distribution with which IR spectra were recorded forms a compromise between low Ar coverage and the mass range over which IR spectra could be recorded. To reduce contamination due to ingrowth from $\mathrm{Cu}_{n}{ }^{+}-\mathrm{Ar}_{m+1}$ complexes, for each cluster size $n$, we display the spectrum for the complex $\mathrm{Cu}_{n}{ }^{+}-\mathrm{Ar}_{m}$ with the highest intensity in the mass spectrum. As a result, spectra for the lower cluster masses have been recorded at relatively high $\mathrm{Ar}$ coverage, illustrated by $\mathrm{Cu}_{4}{ }^{+}-\mathrm{Ar}_{4}$. Its IRMPD spectrum exhibits four well-resolved bands (83, 103, 132, and $207 \mathrm{~cm}^{-1}$ ) with line widths (full width at half-maximum (fwhm)) of $\sim 11 \mathrm{~cm}^{-1}$. To assign this spectrum, it is compared with the calculated spectra of three isomers of $\mathrm{Cu}_{4}^{+}$; comparisons with further isomers can be found in the SI. Only doublet spin isomers are considered here (just as for further even-sized clusters, where singlet states are considered for odd-sized clusters) because they were calculated to be lower in energy than other spin state isomers, in agreement with what has been reported at the LCGTO-GGA theoretical level. ${ }^{21}$ The assignment of the IRMPD spectrum based on the calculated spectra of bare $\mathrm{Cu}_{4}{ }^{+}$is not possible, as it is evident 
that each of the structures proposed does not have sufficient bands to explain the experimental spectrum. Sure enough, the 132 and $207 \mathrm{~cm}^{-1}$ bands match the calculated spectrum for the lowest energy $D_{2 h}$ rhombic structure $4 \mathrm{~A}$ very well, but this leaves the lower frequency bands unaccounted for. The inclusion of the Ar messenger atoms in the DFT calculations quite drastically changes the predicted spectrum. Now all four observed bands are predicted, agreeing in both frequency and relative intensity. The two lowest-frequency bands (observed at 83 and $103 \mathrm{~cm}^{-1}$ ) that were not predicted by the calculations for the bare $\mathrm{Cu}_{4}{ }^{+}$originate from motions of the Ar relative to cluster. Also, slight shifts for the higher frequency bands are calculated, from 130 and $212 \mathrm{~cm}^{-1}$ for the bare clusters to 138 and $215 \mathrm{~cm}^{-1}$ for the complex. These bands are associated with elongations of the cluster along the $\mathrm{Cu}-\mathrm{Cu}$ axes, which are sterically hindered by the presence of the Ar. However, they do not disturb the original structure dramatically, as illustrated by the relatively low calculated binding energies of 0.20 and $0.27 \mathrm{eV}$ for binding to the copper atoms positioned along the long and short axes, respectively. It is of further interest that the presence of $\mathrm{Ar}$ atoms increases the IR intensity of the bands to over ten times, suggesting that the Ar acts as an "antenna”.

The IRMPD spectrum recorded for $\mathrm{Cu}_{5}{ }^{+}-\mathrm{Ar}_{3}$ is simpler than that for $\mathrm{Cu}_{4}{ }^{+}-\mathrm{Ar}_{4}$, exhibiting just two well-resolved bands and suggesting a higher symmetry structure. In the literature, the structure of $\mathrm{Cu}_{5}^{+}$has been the subject of considerable debate, with both planar and 3D structures proposed to be most stable. ${ }^{2,17,22,23}$ Here it is compared with the calculated spectra of three isomers of $\mathrm{Cu}_{5}{ }^{+}$within $0.1 \mathrm{eV}$ relative to each other. The comparison for the bare structures suggests the assignment to a $D_{3 h}$ trigonal bipyramid isomer $5 \mathrm{~A}$, with a reasonable agreement for the bands observed at $188 \mathrm{~cm}^{-1}(177$ $\mathrm{cm}^{-1}$ calculated) and at $121 \mathrm{~cm}^{-1}\left(93 \mathrm{~cm}^{-1}\right.$ calculated). Calculated modes correspond to in-plane and out-of-plane distortions of the triangular base plane. Ar binding to atoms of the triangular base is energetically the most favorable, where the steric hindrance caused by the Ar shifts the calculated bands to 189 and $121 \mathrm{~cm}^{-1}$, respectively, resulting in an almost perfect match with the experimental bands. Ar complexation of the planar structure $5 \mathrm{C}$ is not energetically favorable $(0.06 \mathrm{eV}$ higher than the lowest energy complex of 5A), nor does it provide a better agreement with the observed spectrum. IMS measurements by Weis suggest a planar structure 5C for $\mathrm{Cu}_{5}{ }^{+17}$ Because the current calculations suggest that $\mathrm{Ar}$ complexation energetically favors structure 5A, the current work cannot decisively conclude the discussion on the structure of $\mathrm{Cu}_{5}{ }^{+}$.

The IRMPD spectrum of $\mathrm{Cu}_{7}^{+}-\mathrm{Ar}_{3}$ shows four bands at $103,146,205$, and $225 \mathrm{~cm}^{-1}$, respectively. In contrast with the smaller clusters discussed so far, we can here assign the IRMPD spectrum to a structure based on the calculations for the bare cluster alone: The lowest energy structure $7 \mathrm{~A}$, a pentagonal bipyramid with $D_{5 h}$ symmetry, shows a quite reasonable match with three $\left(102,137\right.$, and $\left.226 \mathrm{~cm}^{-1}\right)$ of four bands found experimentally. Alternative candidate structures $7 \mathrm{~B}$ and 7C, 0.3 and $0.4 \mathrm{eV}$ higher in energy, respectively, are characterized by high-intensity bands around $120 \mathrm{~cm}^{-1}$, which are absent in the IRMPD spectrum. For completeness, we also calculated the spectrum for the lowest energy structures $\mathrm{Cu}_{7}{ }^{+}-$ $\mathrm{Ar}_{3}$ for $7 \mathrm{~A}-\mathrm{C}$. Optimization of $\mathrm{Ar}$ complexes of structure $7 \mathrm{C}$ consistently led to the isomerization of the cluster to either 7A or $7 \mathrm{~B}$. The calculated spectrum for the Ar-complexed $7 \mathrm{~B}$ is essentially the same as that for the bare structure, except for some shifting bands that do not match the experimental spectrum. For 7A, the presence of two Ar atoms on the fivefold symmetry axis does not lead to symmetry breaking, but the third one in the pentagonal plane does, albeit only slightly. As a consequence, extra bands appear in the spectrum, for instance, the high-frequency shoulder of the main band at 150 $\mathrm{cm}^{-1}$, which explains the asymmetric line shape of the observed band. A hint of a band near $70 \mathrm{~cm}^{-1}$ is observed, which would agree with the cluster-Ar vibration predicted. However, because the FEL power is lowest here, a power correction may amplify background noise, and because we do not see the potential band return to the baseline, we cannot draw firm conclusions.

The final system shown is $\mathrm{Cu}_{10}{ }^{+}$. With the increase in cluster size, the number of absorbed $\mathrm{Ar}$ atoms decreases due to the dilution of local charge density. For this reason, structure determination of the $\mathrm{Cu}_{10}{ }^{+}$cluster could be done by employing the photodissociation spectrum of $\mathrm{Cu}_{10}{ }^{+}-\mathrm{Ar}$. The spectrum reflects the growing complexity of the system, showing a rich structure with many overlapping bands. Nevertheless, even on a first comparison with calculated spectra, we are able to assign it to structure 10A, a pentagonal bipyramid, now capped with three $\mathrm{Cu}$ atoms on neighboring faces, making it also interpretable as two interlocked pentagonal bipyramids that share four atoms. With the exception of a weaker band at 213 $\mathrm{cm}^{-1}$, all spectral features are matched. Whereas the $213 \mathrm{~cm}^{-1}$ band could be due to the presence of another isomer, we conclude that the spectrum is by and large dominated by structure 10A. Because of the increase in the system size, the relative influence of the Ar messenger atom on the spectrum is further reduced in comparison with the $\mathrm{Cu}_{7}^{+}$case: DFT calculations for the Ar-tagged system predict an almost identical spectrum as that for the bare cluster, with (apart from a cluster-Ar vibration at $70 \mathrm{~cm}^{-1}$ ) minimal modifications in band frequencies and only a two-fold intensity increase.

In this way, we recorded IRMPD spectra for $\mathrm{Cu}_{n}{ }^{+}(n=3-$ 10) and unambiguously assigned each spectrum to a specific structure. Further experimental and calculated spectra, including spectra for Ar-complexed clusters, can be found in the SI. Beside the clusters discussed above, we find a triangular structure for $\mathrm{Cu}_{3}{ }^{+}$, a capped triangular bipyramid structure for $\mathrm{Cu}_{6}{ }^{+}$, and a pentagonal bipyramid structure capped with one and two $\mathrm{Cu}$ atoms for $\mathrm{Cu}_{8}{ }^{+}$and $\mathrm{Cu}_{9}{ }^{+}$, respectively. It is now of interest to evaluate the stepwise evolution of the cluster structures found, as shown in Figure 2. Clearly, the two smallest clusters $\mathrm{Cu}_{3}{ }^{+}$and $\mathrm{Cu}_{4}{ }^{+}$are planar. However, from $n=$ 5 , all structures found are $3 \mathrm{D}$. What is particularly interesting is that every structure found is formed by the addition of $\mathrm{a} \mathrm{Cu}$ atom onto the structure of the previous cluster size. From $\mathrm{Cu}_{7}{ }^{+}$, the pentagonal motif dominates, forming a template onto which larger clusters form. We thus see no signs of the emergence of $f c c$-like structures, as one would expect from the bulk structure. All structures found here are in agreement with most of the previous theoretical studies, ${ }^{21-26}$ except for the structure of $\mathrm{Cu}_{5}{ }^{+}$discussed above.

When comparing the structures found with those reported from calculations for neutral and anionic copper clusters, it is remarkable that cationic structures undergo the $2 \mathrm{D}$ to $3 \mathrm{D}$ transformation at smaller sizes $(n=5$ for cations and 6 and 7 for anions and neutrals, respectively). ${ }^{24-33}$ The same trend was shown for $\mathrm{Ag}_{n}{ }^{+}(n=6$ versus $n=6 / 7$ (anions/neutrals)) and $\mathrm{Au}_{n}{ }^{+}\left(n=8\right.$ versus 12/11), ${ }^{34}$ illustrating the importance of 


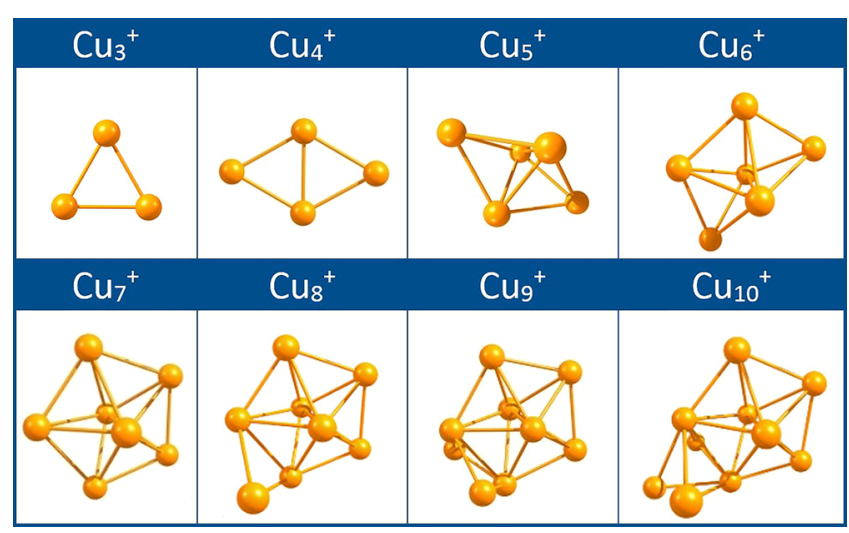

Figure 2. Structures of the small cationic copper clusters $(n=3-10)$ inferred in this work.

charge for the cluster geometry: The structure is a fine balance between interatomic stabilization and surface energies, and as a consequence, the loss of an electron leads to the weakening of the interatomic bonds, and the cluster needs to rearrange to reduce the surface energy. ${ }^{22}$

We can further compare the structures found for $\mathrm{Cu}_{n}{ }^{+}$with those for other coinage metals. Interestingly, for $\mathrm{Ag}_{n}{ }^{+}$, the same structures for the small cluster sizes $(n=3-10)$ are found, except for $n=5 .^{35}$ It is further noteworthy that the structure found for $\mathrm{Cu}_{4}^{+}$is the same as that used for computational studies on deposited $\mathrm{Cu}_{4}{ }^{+}$clusters. Such calculations are thus consistent with the form the clusters had prior to deposition on the $\mathrm{Al}_{2} \mathrm{O}_{3}$ support. ${ }^{7}$

In conclusion, we established the structure of small cationic copper clusters based on a combination of IR photofragmentation spectroscopy of $\mathrm{Cu}_{n}{ }^{+}-\mathrm{Ar}_{m}$ complexes and DFT calculations, which convincingly account for the influence of the Ar.

\section{ASSOCIATED CONTENT}

\section{S Supporting Information}

The Supporting Information is available free of charge on the ACS Publications website at DOI: 10.1021/acs.jpclett.9b00539.

Experimental and computational details, IRMPD spectra of clusters with $n=3-10$ and their comparison to different bare isomers, and the isomers of Ar-tagged complexes of the matching isomer (PDF)

\section{AUTHOR INFORMATION}

\section{Corresponding Author}

*E-mail: j.bakker@ru.nl.

\section{ORCID}

Olga V. Lushchikova: 0000-0002-5699-6818

Lucas Visscher: 0000-0002-7748-6243

Zahra Jamshidi: 0000-0003-0976-1132

\section{Author Contributions}

The manuscript was written through the contributions of all authors. All authors have given approval to the final version of the manuscript.

Notes

The authors declare no competing financial interest.

\section{ACKNOWLEDGMENTS}

This work is part of the Nederlandse Organisatie voor Wetenschappelijk Onderzoek (NWO) Materials for Sustainability program, funded under grant no. 739.017.008. We gratefully acknowledge NWO for the support of the FELIX Laboratory and NWO-EW for computational time on the Cartesius computer cluster (grant 16327). Z.J. acknowledges the Holland Research School for Molecular Chemistry for a fellowship.

\section{REFERENCES}

(1) Knickelbein, M. B. Electric Dipole Polarizabilities of Copper Clusters. J. Chem. Phys. 2004, 120, 10450-10454.

(2) Ichihashi, M.; Corbett, C. A.; Hanmura, T.; Lisy, J. M.; Kondow, T. Size-Specific Reactions of Copper Cluster Ions with a Methanol Molecule. J. Phys. Chem. A 2005, 109, 7872-7880.

(3) Haruta, M. Chance and Necessity: My Encounter with Gold Catalysts. Angew. Chem., Int. Ed. 2014, 53, 52-56.

(4) Kattel, S.; Ramírez, P. J.; Chen, J. G.; Rodriguez, J. A.; Liu, P. Active Sites for $\mathrm{CO}_{2}$ Hydrogenation to Methanol on $\mathrm{Cu} / \mathrm{ZnO}$ Catalysts. Science 2017, 355, 1296-1299.

(5) Behrens, M.; Studt, F.; Kasatkin, I.; Kühl, S.; Hävecker, M.; Abild-Pedersen, F.; Zander, S.; Girgsdies, F.; Kurr, P.; Kniep, B.; et al. The Active Site of Methanol Synthesis over $\mathrm{Cu} / \mathrm{ZnO} / \mathrm{Al}_{2} \mathrm{O}_{3}$ Industrial Catalysts. Science 2012, 336, 893-898.

(6) Natesakhawat, S.; Lekse, J. W.; Baltrus, J. P.; Ohodnicki, P. R.; Howard, B. H.; Deng, X.; Matranga, C. Active Sites and StructureActivity Relationships of Copper-Based Catalysts for Carbon Dioxide Hydrogenation to Methanol. ACS Catal. 2012, 2, 1667-1676.

(7) Liu, C.; Yang, B.; Tyo, E.; Seifert, S.; DeBartolo, J.; von Issendorff, B.; Zapol, P.; Vajda, S.; Curtiss, L. A. Carbon Dioxide Conversion to Methanol over Size-Selected $\mathrm{Cu}_{4}$ Clusters at Low Pressures. J. Am. Chem. Soc. 2015, 137, 8676-8679.

(8) Yang, B.; Liu, C.; Halder, A.; Tyo, E. C.; Martinson, A. B. F.; Seifert, S.; Zapol, P.; Curtiss, L. A.; Vajda, S. Copper Cluster Size Effect in Methanol Synthesis from $\mathrm{CO}_{2}$. J. Phys. Chem. C 2017, 121, 10406-10412.

(9) Powers, D. E.; Hansen, S. G.; Geusic, M. E.; Puiu, A. C.; Hopkins, J. B.; Dietz, T. G.; Duncan, M. A.; Langridge-Smith, P. R. R.; Smalley, R. E. Supersonic Metal Cluster Beams: Laser Photoionization Studies of $\mathrm{Cu}_{2}$. J. Phys. Chem. 1982, 86, 2556-2560.

(10) Leopold, D. G.; Ho, J.; Lineberger, W. C. Photoelectron Spectroscopy of Mass-Selected Metal Cluster Anions. I. $\mathrm{Cu}_{\mathrm{n}}{ }^{-}, \mathrm{n}=1-$ 10. J. Chem. Phys. 1987, 86, 1715-1726.

(11) Taylor, K. J.; Pettiette-Hall, C. L.; Cheshnovsky, O.; Smalley, R. E. Ultraviolet Photoelectron-Spectra of Coinage Metal-Clusters. J. Chem. Phys. 1992, 96, 3319-3329.

(12) Ho, J.; Ervin, K. M.; Lineberger, W. C. Photoelectron Spectroscopy of Metal Cluster Anions: $\mathrm{Cu}_{n}{ }^{-}, \mathrm{Ag}_{n}{ }^{-}$, and $\mathrm{Au}_{n}{ }^{-} . J$. Chem. Phys. 1990, 93, 6987-7002.

(13) Cheshnovsky, O.; Taylor, K. J.; Conceicao, J.; Smalley, R. E. Ultraviolet Photoelectron Spectra of Mass-Selected Copper Clusters: Evolution of the 3d Band. Phys. Rev. Lett. 1990, 64, 1785-1788.

(14) Winter, B. J.; Parks, E. K.; Riley, S. J. Copper Clusters: The Interplay between Electronic and Geometrical Structure. J. Chem. Phys. 1991, 94, 8618-8621.

(15) Jarrold, M. F.; Creegan, K. M. Optical Spectroscopy of Metal Clusters: $\mathrm{Cu}_{4}{ }^{+}$. Chem. Phys. Lett. 1990, 166, 116-122.

(16) Jarrold, M. F.; Creegan, K. M. Photodissociation of Copper Clusters, $\mathrm{Cu}_{\mathrm{n}}^{+}(\mathrm{n}=3-8)$, in the 370-710 nm Wavelength Region. Int. J. Mass Spectrom. Ion Processes 1990, 102, 161-181.

(17) Weis, P. Structure Determination of Gaseous Metal and SemiMetal Cluster Ions by Ion Mobility Spectrometry. Int. J. Mass Spectrom. 2005, 245, 1-13.

(18) Fielicke, A.; Kirilyuk, A.; Ratsch, C.; Behler, J.; Scheffler, M.; Von Helden, G.; Meijer, G. Structure Determination of Isolated Metal 
Clusters via Far-Infrared Spectroscopy. Phys. Rev. Lett. 2004, 93, No. 023401.

(19) Gruene, P.; Rayner, D. M.; Redlich, B.; van der Meer, A. F. G.; Lyon, J. T.; Meijer, G.; Fielicke, A. Structures of Neutral $\mathrm{Au}_{7}{ }^{-}, \mathrm{Au}_{19}{ }^{-}$, and $\mathrm{Au}_{20}{ }^{-}$Clusters in the Gas Phase. Science 2008, 321, 674-676.

(20) Oepts, D.; van der Meer, A. F. G.; Van Amersfoort, P. W. The Free-Electron-Laser User Facility FELIX. Infrared Phys. Technol. 1995, 36, 297-308.

(21) Calaminici, P.; Köster, A. M.; Russo, N.; Salahub, D. R. A Density Functional Study of Small Copper Clusters: $\mathrm{Cu}_{\mathrm{n}}(n<5)$. J. Chem. Phys. 1996, 105, 9546.

(22) Chu, X.; Xiang, M.; Zeng, Q.; Zhu, W.; Yang, M. Competition between Monomer and Dimer Fragmentation Pathways of Cationic $\mathrm{Cu}_{\mathrm{n}}$ clusters of $\mathrm{n}=2-20$. J. Phys. B: At., Mol. Opt. Phys. 2011, 44, 205103-205105.

(23) Guzmán-Ramírez, G.; Aguilera-Granja, F.; Robles, J. DFT and GEGA Genetic Algorithm Optimized Structures of $\mathrm{Cu}_{\mathrm{n}}{ }^{2}(\mathrm{n}= \pm 1,0,2$; $\mathrm{n}=3-13$ ) Clusters. Eur. Phys. J. D 2010, 57, 49-60.

(24) Jug, K.; Zimmermann, B.; Calaminici, P.; Köster, A. M. Structure and Stability of Small Copper Clusters. J. Chem. Phys. 2002, 116, 4497-4507.

(25) Florez, E.; Tiznado, W.; Mondragón, F.; Fuentealba, P. Theoretical Study of the Interaction of Molecular Oxygen with Copper Clusters. J. Phys. Chem. A 2005, 109, 7815-7821.

(26) Chaves, A. S.; Rondina, G. G.; Piotrowski, M. J.; Tereshchuk, P.; Da Silva, J. L. F. The Role of Charge States in the Atomic Structure of $\mathrm{Cu}_{\mathrm{n}}$ and $\mathrm{Pt}_{\mathrm{n}}(\mathrm{n}=2-14$ Atoms) Clusters: A DFT Investigation. J. Phys. Chem. A 2014, 118, 10813-10821.

(27) Aakeby, H.; Panas, I.; Pettersson, L. G. M.; Siegbahn, P.; Wahlgren, U. Electronic and Geometric Structure of the $\mathrm{Cu}_{\mathrm{n}}$ Cluster Anions $(n \leq 10)$. J. Phys. Chem. 1990, 94, 5471-5477.

(28) Padilla-Campos, L. Theoretical Investigation of the Adsorption of Oxygen on Small Copper Clusters. J. Mol. Struct.: THEOCHEM 2007, 815, 63-69.

(29) Poater, A.; Duran, M.; Jaque, P.; Toro-Labbé, A.; Solà, M. Molecular Structure and Bonding of Copper Cluster Monocarbonyls $\mathrm{Cu}_{\mathrm{n}} \mathrm{CO}(\mathrm{n}=1-9)$. J. Phys. Chem. B 2006, 110, 6526-6536.

(30) Jaque, P.; Toro-Labbé, A. Characterization of Copper Clusters through the Use of Density Functional Theory Reactivity Descriptors. J. Chem. Phys. 2002, 117, 3208-3218.

(31) Guvelioglu, G. H.; Ma, P.; He, X.; Forrey, R. C.; Cheng, H. Evolution of Small Copper Clusters and Dissociative Chemisorption of Hydrogen. Phys. Rev. Lett. 2005, 94, 3-6.

(32) Jackson, K. A. First-Principles Study of the Structural and Electronic Properties of $\mathrm{Cu}$ Clusters. Phys. Rev. B: Condens. Matter Mater. Phys. 1993, 47, 9715-9722.

(33) Fernández, E.; Boronat, M.; Corma, A. Trends in the Reactivity of Molecular $\mathrm{O}_{2}$ with Copper Clusters: Influence of Size and Shape. J. Phys. Chem. C 2015, 119, 19832-19846.

(34) Fernández, E. M.; Soler, J. M.; Garzón, I. L.; Balbás, L. C. Trends in the Structure and Bonding of Noble Metal Clusters. Phys. Rev. B: Condens. Matter Mater. Phys. 2004, 70, 165403-165416.

(35) van der Tol, J.; Jia, D.; Li, Y.; Chernyy, V.; Bakker, J. M.; Nguyen, M. T.; Lievens, P.; Janssens, E. Structural Assignment of Small Cationic Silver Clusters by Far-Infrared Spectroscopy and DFT Calculations. Phys. Chem. Chem. Phys. 2017, 19, 19360-19368. 